\title{
Tipificación de Aporosella hassleriana Chodat (Phyllanthaceae) de la flora del Paraguay
}

\author{
Lorenzo Ramella \& Fernando O. Zuloaga
}

\begin{abstract}
RAMELLA, L. \& F.O. ZULOAGA (2017). Typification of Aporosella hassleriana Chodat (Phyllanthaceae) of the flora of Paraguay. Candollea 72: 403-404. In Spanish, English and Spanish abstracts. DOI: http://dx.doi.org/10.15553/c2017v722a16

The lectotype specimen of Aporosella hassleriana Chodat (Phyllanthaceae) is designated from the Hassler's collections kept at $\mathrm{G}$.

\section{Resumen}

RAMELLA, L. \& F.O. ZULOAGA (2017). Tipificación de Aporosella hassleriana Chodat (Phyllanthaceae) de la flora del Paraguay. Candollea 72: 403-404. En español, resúmenes en inglés y español. DOI: http://dx.doi.org/10.15553/c2017v722a16

El lectótipo de Aporosella hassleriana Chodat (Phyllanthaceae) está designado entre las colecciones de Hassler conservadas en $\mathrm{G}$.
\end{abstract}

\section{Keywords}

PHYLLANTHACEAE - Euphorbiaceae - Aporosella - Typification - Paraguay 


\section{Introducción}

Durante la preparación de la familia Phyllanthaceae para la Flora Argentina (Zuloaga \& Belgrano, en preparación), ha surgido la necesidad de tipificar el nombre Aporosella hassleriana Chodat. Este nombre ha sido descrito a partir de dos síntipos colectados por Emil Hassler en Paraguay y es también el tipo del género Aporosella Chodat.

\section{Tipificación}

Aporosella hassleriana Chodat in Bull. Herb. Boissier ser. 2, 5: 489. 1905.

Lectotypus (in hoc loco designatus): Paraguay. Concepción: "Prope Concepcion in insula Chaco y ad ripam fluminis”, VIII.1901-1902, Hassler, E. 7161 (G [G00229904]!; isolecto-: G [G00229906, G00229907, G00229911, G00229912]!, MICH [MICH1107950]!, MO [MO260328]!, P [P00608946, P00608947, P00608949]!, S [S-R-10484]!, UC [UC941632]!).

Syntypus: ParaguaY. Concepción: "Prope Concepcion ad ripam fluminis Paraguay”, IX.1901-1902, Hassler, E. 7161 a (G [G00229905]!; isosyn-: G [G00229908, G00229909, G00229910, G00229913]!, K [K000573221]!, MPU [MPU014789, MPU014790]!, NY [NY00246236]!).

Notas. - El lectótipo designado aquí es el material utilizado por Chodat en las descripciones tanto del género Aporosella como de $A$. hassleriana: una serie de ilustraciones analíticas de la morfología floral dibujadas por Chodat son parte del espécimen elegido.

\section{Referencia}

Zuloaga, F.O. \& M. Belgrano (en preparación). Phyllanthaceae. Fl. Argentina 17. 